\title{
El arte como instrumento para aprender a sobrellevar la incertidumbre
}

\author{
Art as an instrument to learn how to overcome uncertainty
}

\begin{abstract}
La incertidumbre es una posición incómoda, pero la certeza es una posición absurda.
\end{abstract}

Voltaire (1694 - 1778)

Continuamos con la serie de artículos relacionados con el arte como instrumento educativo en medicina. El objetivo fue introducir el arte como recurso docente en la formación de los profesionales de la salud, propuesta basada en la capacidad que el lenguaje simbólico del arte tiene de reflejar la dimensión inefable humana. ${ }^{1}$

En el presente artículo analizaremos otro de los aspectos fundamentales de la labor médica que puede ser comprendido con mayor profundidad a través del arte: la naturaleza de la incertidumbre y su manejo. La incertidumbre es la falta de certeza inherente al azar de la vida, a la cuál por supuesto tampoco escapa la labor médica. No debe ser confundida con la mera duda, pues mientras en la duda hay ausencia de acción por temor a lo desconocido, en la incertidumbre se evalúa la situación y se opta por la alternativa más favorable, aun a sabiendas de los imponderables que la pueblan, pero se decide manejarlos a través de una continua reevaluación de la situación y reajuste del curso de acción originalmente planeado. Otra de las funciones del profesional de la salud es la de ayudar al paciente a encauzar su incertidumbre, de lo cual sólo será capaz si previamente ha logrado tolerar la propia. ${ }^{2-5}$

Por otra parte, resulta entendible que si la ausencia de completitud afecta al ideal mundo matemático (Gödel) y la indeterminación al diminuto mundo cuántico (Heisenberg), la incertidumbre impregne entonces a nuestro complejísimo (caótico) mundo supra-atómico. ${ }^{6}$

Desde el arte estos conceptos se exponen con suma claridad, por ejemplo en el poema "De todo quedaron tres cosas" del gran escritor Fernando Sabino. ${ }^{7}$ En esta obra, el autor Pessoa no sólo nos habla de la inexorable y permanente presencia de la incertidumbre en nuestras vidas, sino además de cómo su fórmula de manejo consiste en una amalgama de paciencia, aceptación y voluntad de reinicio sin fin. Veamos el poema:

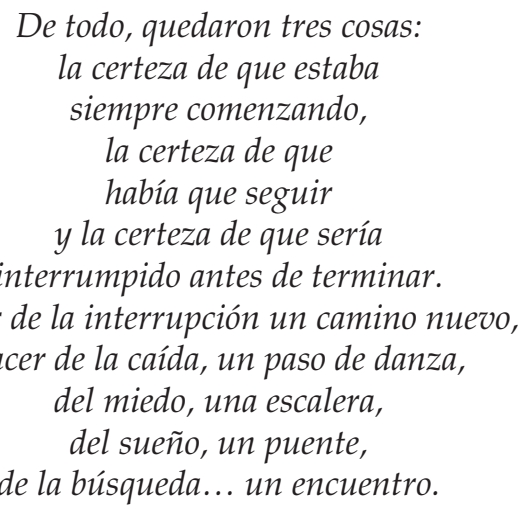

Concluimos entonces lo valioso que resulta la incorporación del arte como un instrumento educativo en pos de lograr una mayor compresión de cuál es la naturaleza de la incertidumbre, así como de lograr la fórmula para su manejo por parte de los profesionales de la salud.

Dr. Carlos G. Musso y Dra. Paula A. Enz Escuela de Medicina, Instituto Universitario del Hospital Italiano de Buenos Aires. Argentina

http:/ /dx.doi.org/10.5546/aap.2015.485

\section{REFERENCIAS}

1. Musso CG, Enz PA. El arte como instrumento educativo en medicina. Arch Argent Pediatr. 2014;112(6):494-5.

2. Castaneda C. La rueda del tiempo: los chamales del antiguo México y sus pensamientos acerca de la vida, la muerte y el universo. Madrid: Gaia, 1998.

3. Ferry L. La sabiduría de los mitos: aprender a vivir. Buenos Aires: Taurus, 2010.

4. Musso CG, Enz P. Arte y naturaleza humana VII. Rev Hosp Ital B. Aires. 2012;32:(1).

5. Musso CG, Enz P. Arte y naturaleza humana X. Rev Hosp Ital B. Aires. 2012;32(4):194-5.

6. MussoCG. Obras maestras del arte universal y la medicina: Muerte y hombre (Los autovidentes II) de Egon Schiele (18901918). Evid actual práct ambul. (en prensa) 2015.

7. Sabino F. Encuentro marcado. Barcelona: Luis de Caralt, 1964. 


\section{Humanismo y rigurosidad científica: gemelos separados al nacer Humanism and scientific rigor: twins separated at birth}

El humanismo médico y la rigurosidad científica son complementarios, no opuestos; especialmente en nuestra profesión. Cualquier médico, sin importar su edad ni años de práctica, seguramente estará de acuerdo. Sin embargo, probablemente también experimentará desequilibrios a la hora de practicarlos.

El refugio en un cientificismo dogmático quizá tenga su origen en la ignorancia no sólo de la importancia de los valores que regulan la conducta del médico sino también, o principalmente, en la ignorancia de cómo fomentarlos.

¿Cómo brindar conocimientos humanísticos que luego se transubstancien en aptitudes concretas frente al paciente y frente a colegas? Diversas instituciones realizan heroicos esfuerzos en pos de brindar el ambiente y el tiempo necesarios para prevenir el síndrome de desgaste profesional. Muchas de las actividades que proponen se basan en una práctica más humanizada de la medicina. ${ }^{1}$

Más tarde o más temprano la balanza de la práctica se desequilibra hacia el platillo de la formación científica y del acopio de "know how". Pero simplemente se busca el éxito médico con las herramientas que se tienen; no necesariamente con las mejores. La actualización constante, la vida académica, la consideración de nuevas prácticas, el desarrollo del pensamiento crítico, etc., son necesarios pero no suficientes para el éxito en la atención médica (no para un éxito integral, que incluya la plena satisfacción del médico consigo mismo como ser humano y no sólo con la labor técnica realizada).

Han aparecido estudios donde se empieza a demostrar lo que la sabiduría popular afirma desde siempre: que el éxito no lleva a la felicidad ${ }^{3}$, al mismo tiempo que sentir mayor felicidad lleva a ser más eficaz y más responsable en las tareas realizadas. De ahí que se esté invirtiendo en brindar a los trabajadores compensaciones que aspiran a aportar algo a su felicidad. De todos modos, auguro que en no demasiado tiempo se comprobará que todo lo externo que se brinde (y que en nuestra profesión son factores de protección para el burn out), no va a alcanzar, ya que la felicidad no depende de lo que se tiene sino de lo que se es. En ese ser se puede invertir para que la alegría y la satisfacción con uno mismo sean la base para un trabajo que equilibre el humanismo y la formación científica.

La empatía, la capacidad de brindar afecto, la consideración, son de un valor incalculable a la hora de atender al paciente y a sus familiares. Gobernar las contrariedades o las molestias, ser capaz de tener un compromiso real con el paciente sin que implique un compromiso completo o inadecuado del ánimo, son también elementos que aportan al éxito externo y al interno, aportándonos mayor satisfacción y bienestar. Estas y otras aptitudes que no se relacionan específicamente con la resolución del problema médico, pueden llevar a experimentar gran bienestar antes, durante y luego de la atención de un niño, así como del contacto con sus padres.

Nuestra naturaleza humana se desequilibra cuando atendemos al paciente con un solo polo. La tarea que realizamos requiere tanto del trato humanitario como de la rigurosidad científica. Una atención médica desequilibrada nos perjudica y perjudica a los pacientes pero, ¿cómo mejorar lo que se es como ser humano?, ¿en dónde se fragua el equilibrio buscado? En la mente. La educación de la propia mente es un paso fundamental para el cultivo sistemático, consecuente y armónico del humanismo en uno mismo. Se ha presentado como un desiderátum desde hace muchísimo tiempo, como recuerda el pediatra y psiquiatra Daniel Siegel: «Hace más de 100 años, el padre de la psicología moderna, Williams James (1890-1981), dijo que la facultad de volver a encauzar una y otra vez la atención que divaga sería "la educación por excelencia". También dijo que el problema es que no sabemos cómo hacerlo». ${ }^{3}$ Han ocurrido avances desde entonces. Diversas disciplinas brindan claves en este sentido, entre la cuales se encuentra una argentina: la Logosofía. En 1956, el Dr. Friedenthal, quien había practicado la disciplina logosófica desde muy joven, escribió: "Los conocimientos que se traducen en el arte de curar tienen indudablemente que transitar por la estructura psicológica de quien los aplica. Alli, en ese contacto intimo, profundo, en que se encastran ciencia 
y carácter, reciben influencias benéficas como también negativas y hasta pueden desnaturalizarse en su más noble esencia (...). De ahi que todo profesional del arte de curar deba ser un celoso guardián de la salud de su propia alma, pues la sociedad le ha conferido una altísima responsabilidad que deberá administrar conscientemente si es que quiere gozar de legítima tranquilidad espiritual". ${ }^{4}$ Es en la mente del médico donde confluyen la información y la formación médica; es también allí donde pueden florecer los valores y virtudes que permitan una práctica más humana.

Avanzar en pos de que la mente responda a las propias intenciones y aspiraciones (en vez de en forma automática y muchas veces contraria a nuestros propósitos más profundos) es un paso fundamental para que las conductas elevadas sean una realidad sistemática y no aleatoria. Esto es apenas una parte del proceso de superación integral que se puede encarar. En este constate proceso de superación interna (constante por la perseverancia en el esfuerzo, no porque todos los intentos sean exitosos...), implica avanzar en la realización de un nuevo humanismo que "es el ser racional y consciente realizando en sí mismo las excelencias de su condición de humano y de su contenido espiritual sobre la base de una incesante superación. Dichas excelencias deberán trascender por el ejemplo y la enseñanza a toda la humanidad". ${ }^{5}$

Como ya se dijo, todos los recursos que usemos para lograr una vida más consciente nos llevarán a ser más rigurosos en la obtención de conocimientos científicos y a querer perfeccionarnos también en ese campo. Una vida en la que trabajemos diariamente contra nuestras modalidades negativas; en la que aprendamos a dejar brotar la alegría de estar vivos; en la que sea un objetivo cotidiano la obtención de conocimientos superiores; en la que encontremos satisfacción por servir al semejante.
Pienso que en medicina la "genética" de la rigurosidad científica y del humanismo incluye los mismos elementos: hacer el bien, curar, aliviar. Sin embargo, como puede ocurrir con los hermanos gemelos, ambos aspectos pueden seguir caminos diferentes que terminen en el dogmatismo, en la falta de comunicación entre uno y otro. En nosotros mismos radica la capacidad de encontrar las herramientas para conciliarlos, para ver unidad donde antes hubo separatismo. Una de las mejores herramientas es el conocimiento de uno mismo y la superación resultante.

Parafraseando al Dr. Escardó: no se puede ser mejor médico de lo que se es como persona. Así, la práctica de una disciplina de superación que nos haga mejores seres humanos, nos hará también mejores médicos. El enunciado de la SAP "un niño sano en un mundo mejor" puede ir convirtiéndose en realidad mejorando la parte más cercana del mundo: nosotros mismos.

\section{Dra. Paula A. Pradines \\ Sanatorio "Franchin" pradines.paula@gmail.com}

http:/ /dx.doi.org/10.5546/aap.2015.486

\section{REFERENCIAS}

1. Catsicaris C, Eymann A, Cacchiarelli N, Usandivaras I. La persona del médico residente y el síndrome de desgaste profesional (burnout).Un modelo de prevención en la formación médica. Arch Argent Pediatr 2007;105(3):236-40.

2. Gilbert DT. Ordinary personology. In: Gilbert D, Fiske S, Lindzey G (Eds.), The handbook of social psychology. 4th ed, Vol 2. New York: Random House, 1998: 89-150.

3. Siegel DJ. Mindfulness y psicoterapia. Barcelona: Paidos. 2012:55.

4. Friedenthal M. Psicología para los profesionales del arte de curar. $2^{\circ}$ ed. Buenos Aires: Progental, 1956:3.

5. González Pecotche CB. Mecanismo de la vida consciente, $7^{\circ}$ ed. Buenos Aires: Logosófica, 2014:106.

\section{Desarrollo pulmonar en niños y su asociación con la polución ambiental Lung development in children and its relationship to environmental pollution}

La polución ambiental produce diferentes efectos deletéreos para la salud, principalmente para el sistema respiratorio. En general, las ciudades con una mayor densidad poblacional y una gran actividad industrial generan un mayor enrarecimiento del medioambiente debido a la producción aumentada de contaminantes por la combustión de motores tanto de automóviles como de grandes fábricas.

El vivir en una ciudad con un alto nivel de 
polución ambiental lleva a que las partículas de diferentes tamaños suspendidas en el medioambiente sean inhaladas por los habitantes del lugar, lo que produce un gran número de patologías, especialmente respiratorias. Los niños, que se encuentran en crecimiento y desarrollo permanente, no están exentos de ser afectados por estas noxas externas, que pueden producir diferentes síntomas respiratorios y afectar el normal crecimiento y desarrollo pulmonar. El crecimiento de las urbanizaciones va acompañado del aumento de la polución ambiental, lo cual aumenta exponencialmente el desarrollo de asma como enfermedad y de sus exacerbaciones. Un estudio en 10 ciudades europeas mostró que, en los niños con asma, el 15\% de las exacerbaciones se atribuyeron a polución relacionada con el tráfico automovilístico cercano a las rutas. ${ }^{8}$

Existen mecanismos de acción directos e indirectos para producir alteraciones pulmonares. En los países con ciudades muy grandes, como China, las partículas del medioambiente afectan directamente el epitelio y los neurorreceptores de las vías aéreas y producen irritación e inflamación. En los países desarrollados, en donde hay políticas de control sobre la producción de contaminación ambiental y presentan más bajas concentraciones de polutantes, existen partículas finas y ultrafinas que producen inflamación (ozono, dióxido de nitrógeno y materia particulada $<2,5$ ) y otras que producen hiperreactividad (ozono y dióxido de nitrógeno), presentes estos dos componentes en el asma bronquial. ${ }^{10,11}$ Todas estas partículas también han sido asociadas a la producción de estrés oxidativo, lo cual es una característica del asma grave, aunque no es claro su mecanismo de producción. ${ }^{12}$

W. J. Gauderman y col., evaluaron si existía una asociación entre la calidad del aire respirado y el desarrollo pulmonar de los niños en varias ciudades del sur de California, Estados Unidos. ${ }^{1}$ En las últimas décadas, en California, se implementaron enérgicas políticas de control de la polución ambiental para poder disminuir la contaminación ambiental y mejorar la calidad del medioambiente. Ellos evaluaron la función pulmonar con espirometrías realizadas anualmente a 2120 niños de entre 11 y 15 años de 3 cohortes diferentes, 1994-1998, 1997-2001 y 2007-2011, y vieron el crecimiento en esos cuatro años del volumen espiratorio forzado al primer segundo (forced expiratory volumen in 1 second; $\mathrm{FEV}_{1}$, por sus siglas en inglés) y de la capacidad vital forzada (forced vital capacity; FVC, por sus siglas en inglés) y lo asociaron con la disminución de la contaminación ambiental. ${ }^{1}$

Durante los 4 años que se evaluaron las 3 cohortes, encontraron una mejoría en $\mathrm{FEV}_{1} \mathrm{y}$ FVC a medida que disminuyeron los niveles de dióxido de nitrógeno y la disminución de la presencia de partículas de 2,5 y $10 \mu \mathrm{m}$ de diámetro aerodinámico. Esta mejoría de la función pulmonar se observó tanto en varones como en mujeres y en asmáticos como en no asmáticos. Esta asociación fue más importante para el aumento del $\mathrm{FEV}_{1}(91,4 \mathrm{ml})$ en los cuatro años, en relación con la disminución de los niveles de dióxido de nitrógeno (14,1 ppb), $\mathrm{p}<0,0001$, que no se observó para la disminución del ozono. La proporción de niños con un baja función pulmonar (<80\% del predicho para $\mathrm{FEV}_{1}$ y FVC) fue claramente menor en la cohorte de 2007 a $2011(3,6 \%)$, la cual estuvo menos expuesta a contaminantes ambientales, comparada con las otras cohortes (7,9\% y 6,3\%).

El crecimiento pulmonar en niños se produce a una gran velocidad desde el nacimiento y durante los primeros 6-8 meses de vida. Este proceso continúa siendo importante hasta los dos años de edad, en los que sufre una gran desaceleración, a pesar de seguir aumentando el número de alvéolos hasta los 8-10 años de edad. Luego, con el crecimiento somático de los adolescentes, se observa un aumento del tamaño pulmonar, pero sin aumentar el número de alvéolos. ${ }^{2,3}$

En el trabajo de Gauderman y col., basado en una exitosa política del estado de California, EE.UU., de disminuir los niveles de contaminación ambiental, y encontrando una mejoría de la función pulmonar en estos grupos de adolescentes de distintos lugares del sur de California, luego de corregir los datos analizados por distintas posibles variables confundidoras, no se presenta un grupo control de una comunidad en donde no se haya implementado esta política de reducción de los niveles de polución ambiental. Los adolescentes presentan un aumento del tamaño pulmonar basado en el crecimiento somático, que en esa edad, muchas veces, está acompañado de una intensa actividad física, la cual mejora la capacidad aeróbica. Un grupo control de la misma edad nos aseguraría que el hallazgo de la mejoría de la función pulmonar se debe a la mejoría de los niveles de contaminación ambiental y no a un crecimiento pulmonar y somático normal. A pesar de esto, y observándose un incremento de la función pulmonar a los 15 años comparado con la basal medida a los 
11 años en cada una de las cohortes (la cohorte de 2007 a 2011 estuvo expuesta a una menor contaminación ambiental, ya que había en ese período una marcada disminución de polución en comparación con las otras cohortes que fueron evaluadas previamente), se presenta una mejor función pulmonar basal y un menor número de adolescentes con una función pulmonar $\left(\mathrm{FEV}_{1}\right.$ y FVC) basal menor del $80 \%$. Esto nos lleva a inferir que hubo un efecto positivo de la política implementada en California para disminuir los niveles de contaminantes ambientales y mejorar la calidad del aire que se respira, la cual produjo una mejoría en el crecimiento y desarrollo pulmonar de los niños expuestos. Este estudio no encontró una asociación con la exposición al ozono, que, en un reciente trabajo del Centers for Desease Control and Prevention (CDC), muestra una asociación positiva en muertes por patologías respiratorias en pacientes expuestos a mayores niveles de ozono. ${ }^{4}$

El impacto que presentan los altos niveles de polución ambiental sobre la salud respiratoria de los niños en constante crecimiento afecta el desarrollo pulmonar y aumenta la morbilidad de diferentes patologías pulmonares. Se puede ver con claridad en estos pocos estudios cómo políticas de Gobiernos sobre el control de la emisión de polutantes ambientales han mejorado sustancialmente el desarrollo pulmonar en niños y han disminuido las patologías respiratorias.

En un mundo en constante desarrollo con aumento de industrias y tráfico vehicular como los mayores generadores de polución ambiental, es posible sostener este crecimiento con un control adecuado de la emisión de polutantes para poder brindar un sano crecimiento y desarrollo de nuestros niños.
Dr. Conrado J. Llapur

Hospital del Niño Jesús de Tucumán,

Facultad de Medicina,

Universidad Nacional de Tucumán.

http:/ /dx.doi.org/10.5546/aap.2015.487

\section{REFERENCIAS}

1. Gauderman WJ, Urman R, Avol E, Berhane K, et al. Association of improved air quality with lung development in children. N Engl J Med 2015;372(10):905-13.

2. Hislop AA, Wigglesworth JS, Desai R. Alveolar development in the human fetus and infant. Early Hum Dev 1986;13(1):1-11.

3. Hislop A, Reid L. Development of the acinus in the human lung. Thorax 1974;29(1):90-4.

4. Hao Y, Balluz L, Strosnider H, Wen XJ, Li C, Qualters JR. Ozone, Fine Particulate Matter and Chronic Lower Respiratory Disease Mortality in the United States. Am J Respir Crit Care Med 2015; May 27. [Epub ahead of print]

5. Gauderman WJ, Avol E, Gilliland F, Vora H, et al. The effect of air pollution on lung development from 10 to 18 years of age. N Engl J Med 2004;351(11):1057-67.

6. Elliot J, Vullermin P, Robinson P. Maternal cigarette smoking is associated with increased inner airway wall thickness in children who die from sudden infant death syndrome. Am J Respir Crit Care Med 1998;158(3):802-6.

7. Tepper RS, Williams-Nkomo T, Martinez T, Kisling J, et al. Parental smoking and airway reactivity in healthy infants. Am J Respir Crit Care Med 2005;171(1):78-82.

8. Perez L, Declercq C, Iñiguez C, Aguilera I, et al. Chronic burden of near-roadway traffic pollution in 10 European cities (APHEKOMnetwork). Eur Respir J 2013;42(3):594-605.

9. Brunst KJ, Ryan PH, Brokamp C, Bernstein D, et al. Timing and Duration of Traffic-Related Air Pollution Exposure and the Risk for Childhood Wheeze and Asthma. Am J Respir Crit Care Med 2015; Jun 24. [Epub ahead of print]

10. Aris RM, Christian D, Hearne PQ, Kerr K, et al. Ozoneinduced airway inflammation in human subjects as determined by airway lavage and biopsy. Am Rev Respir Dis 1993;148(5):1363-72.

11. Solomon C, Christian DL, Chen LL, Welch BS, et al. Effect of serial-day exposure to nitrogen dioxide on airway and blood leukocytes and lymphocyte subsets. Eur Respir J 2000;15(5):922-8.

12. Liu L, Poon R, Chen L, Frescura AM, et al. Acute effects of air pollution on pulmonary function, airway inflammation, and oxidative stress in asthmatic children. Environ Health Perspect 2009;117(4):668-74. 